\title{
Longevidade e Sexualidade: uma Abordagem Inerente à Atuação do Enfermeiro Enquanto Educador em Saúde
}

\section{Longevity and Sexuality: an Inherent Approach in the Nursing Practice as a Health Educator}

\author{
Maria Elizabeth da Costa Felipe Santiago
}

\begin{abstract}
Centro Universitário das Faculdades Metropolitanas Unidas, Pós-Graduação Lato Sensu em Enfermagem do Trabalho. RN, Brasil.
\end{abstract} E-mail: elizabeth55_@hotmail.com

\begin{abstract}
Resumo
Falar da sexualidade na terceira idade é uma temática ainda ligada a tabus, pois há escassez de menções e diálogos tanto pelos profissionais de saúde como pelos próprios idosos que se intimidam e deixam de praticar a sexualidade pela repressão da sociedade, assim como pela complexidade oriunda de alterações fisiológicas e psíquicas, que também colaboram como grandes fatores para não prática da atividade sexual. Todavia, esse envolvimento de amor, afeto e contato físico estabelece um vínculo de laços prazerosos que precisa ser dialogado no decurso de todas as etapas da vida. Assim sendo, este estudo tem por objetivo analisar a produção científica sobre a sexualidade na terceira idade e os problemas enfrentados por essa população, o que engloba e requer dos profissionais da saúde, em especial o enfermeiro como educador nesse processo de envelhecimento e sexualidade, em que o presente artigo se trata de uma revisão narrativa realizada através da Biblioteca Virtual em Saúde e consultas aos bancos de dados ScientificElectronic Library Online (SciELO), Literatura Latino-Americana e do Caribe em Ciências da Saúde (Lilacs) e na Medical LiteratureAnalysisandRetrieval System Online (MEDLINE). Como critérios de inclusão foram aplicados: artigos com disponibilidade completa e de origem nacional, bem como relação direta com o estudo. À vista disso é possível levar em consideração o envelhecimento enfrentado positivamente, a prática sexual que aceita, religião e a educação, pois estes são responsáveis pela influência do exercício sexual, devendo ser encorajados por meio de estímulo visual, carícias e afeto, a atividade sexual continua sendo exercida caso o próprio idoso e seu parceiro tenham condições físicas e mentais para permanecerem executando. Visto isso, a sexualidade perdura em construção no decorrer do trajeto do ser humano, e em face desse processo o enfermeiro deve exercer o papel de educador, incluindo o ensino em saúde no ambiente de atuação profissional, no que se refere à educação sexual.
\end{abstract}

Palavras-chave: Terceira Idade. Profissionais de Enfermagem. Educação Sexual.

\begin{abstract}
Talking about sexuality in the third age is a topic still related to taboos, because there is a shortage of mentions and dialogues both by health professionals and by the elderly themselves who are intimidated and stop practicing sexuality due to the repressing society, as well as by the complexity of physiological changes and psychic factors that also collaborate as great factors for non-practice of sexual activity. However, this involvement of love, affection and physical contact establishes a bond of pleasurable bonds that needs to be dialogued throughout all the stages of life. Therefore, this study aims to analyze the scientific production on sexuality in the third age and the problems faced by this population, which encompasses and requires from the health professionals, especially the nurse as educator in this process of aging and sexuality, when this article deals with a narrative review carried out through the Virtual Health Library and consultations with the ScientificElectronic Library Online (SciELO), Latin American and Caribbean Literature in Health Sciences (Lilacs) and the Medical Literature Analysis andRetrieval System Online (MEDLINE), being followed as inclusion criteria: articles with complete availability and of national origin, as well as direct relationship with the study. Therefore, it is possible to take into account aging faced positively, the sexual practice which accepts religion and education, as they are responsible for the sexual exercise influence, these should be encouraged through visual stimulation, caresses and affection, activity continue to be exercised if the elderly and his or her partner have physical and mental conditions. Thus,, sexuality continues to be built during the course of the human being, and in the face of this process, the nurse must exercise the role of educator, including health education in the professional environment, regarding sex education.
\end{abstract}

Keywords: Elderly. Nursing Professionals. Sex Education.

\section{Introdução}

A população brasileira vem envelhecendo, como pode ser verificado pela transcrição demográfica e pela diminuição das taxas de mortalidade e fecundidade (RODRIGUES et al., 2012). A somatória dessas duas razões influencia no envelhecimento global, com as pessoas vivendo mais tempo. Do contrário, há diminuição nos casos de nascimento. Para a Organização Mundial de Saúde (OMS), a terceira idade em países em desenvolvimento é formada por pessoas a partir dos 60 anos, e em países desenvolvidos, a partir dos 65 anos
(MENDES et al., 2005).

Segundo Mendes et al. (2005), envelhecer é um processo natural que caracteriza uma etapa da vida do homem e ocorre por mudanças físicas, psicológicas e sociais que acometem, de forma particular, cada indivíduo com sobrevida prolongada.

Mesmo que a velhice seja algo relativo, e que seu início resulte da disposição, iniciativa, interesse e independência de cada indivíduo em conformidade, há particularidade em que cada um leva em consideração a qualidade de vida e seu estado de saúde (BRASIL, 2005). O envelhecer não 
deve expressar a imagem de debilitação, infelicidade ou ser assexuado. Muito embora os nossos comportamentos culturais transmitam mensagens diversificadas de mitos e posicionamento na sociedade de que os seres humanos em idade desenvolvida, particularmente no que diz respeito aos assuntos relacionados à sexualidade, são vistos como obstáculo quanto à demonstração nessa etapa de suas vidas (GRANDIN; SOUSA; LOBO, 2012). Segundo os autores, as ressonantes a respeito do envelhecimento sobre a sexualidade estabelecem conteúdos peculiares e sobrecarregados de preconceitos, em que por meio das mudanças físicas, que mesmo sendo naturais e comuns podem influenciar no comportamento, na resposta sexual e nas questões da sexualidade no envelhecimento.

Sexualidade é uma identidade que expressa como a pessoa estabelece a relação consigo e com os demais, e está presente nos seres humanos a partir da vida intrauterina até o instante de morte. Não há que temer a idade como causa de redução do prazer sexual. Existem mudanças na resposta sexual qualitativa e quantitativa no decorrer da idade, essas alterações não se distinguem do contexto orgânico, pois da mesma forma são alteradas pelo tempo, como: locomoção, digestão e circulação (SOUZA, 2010).

No sexo masculino, por intermédio de outras alterações na atividade sexual, as ereções espontâneas não ocorrerão em igual rapidez e simplicidade, além de perderem parte da consistência da juventude. No gênero feminino, apesar de as mudanças ocorrerem em longo prazo, a mulher vivencia a diminuição do hormônio sexual, o estrogênio, no momento da menopausa, percorrendo períodos de extremo incômodo, podendo apresentar manifestações emocionais como ansiedade e estresse; e físicas, como calor, paredes vaginais podendo se tornar delgadas e lisas, acarretando atrofia da mucosa vaginal e diminuição de sua lubrificação. Já em outras situações, o que vai interferir são as causas psicológicas e/ou culturais (ROSENTHAL, 2014).

Todas essas transformações inevitáveis do envelhecimento não influenciarão fatalmente no prazer masculino e feminino, mesmo com essas alterações, os idosos poderão ser capazes de aproveitar a relação sexual e o próprio ato sexual pode se constituir como experiência sensual e prazerosa (GRANDIN; SOUSA; LOBO, 2012).

Ao concordar que o envelhecer não se caracteriza apenas como uma fase de perdas, mais também de ganhos, em que se adquire um olhar mais amplo a respeito do desenvolvimento da sexualidade, reconhecendo que o prazer carregado do contato físico e afetivo com o companheiro pode aumentar e completar as satisfações sexuais do casal, pois, com o passar do tempo, o homem e a mulher são favorecidos pelo autoconhecimento e o conhecimento mais íntimo do outro. Tendo em vista que a relação do casal tende a ser mais valorizada pelas particularidades e não pela quantidade de ereções, orgasmos e vivência prazerosa, vão além do ato sexual em si (DUARTE; DHIOGO, 2010).
Tratar da sexualidade na terceira idade é se ajustar a um assunto que exala novos descobrimentos, que se expandem a cada dia, mesmo que ainda se encontre cercado de tantos tabus, seja por parte dos jovens, dos idosos e dos profissionais, até mesmo daqueles que atuam na área da gerontologia (PAPALÉO NETTO, 2010). Segundo o autor, ao se interessar pela temática, é interessante questionar-se: como anda nossa própria vida sexual? Aque princípios morais ou prejulgamentos estamos submetidos? O que se compreende por sexualidade? Como enfrentamos nosso próprio envelhecer?

Diante do exposto, a finalidade na escolha da temática vem em decorrência do novo perfil da população, que necessita de estudos com o propósito de discutir o desenvolvimento desses indivíduos que constituem hoje uma grande parcela da população, o que requer cuidados específicos e direcionados.

Em face dessa temática, sexualidade na terceira idade, pode-se constituir um assunto pouco abordado e explorado, pois os idosos e os profissionais de saúde mostram certo receio na conversação sobre sexualidade na longevidade, visto que grande parte destes ainda possui concepção ultrapassada, reflexo da educação repressora recebida no passado e, por consequência, produzindo muitas dúvidas, em fazer ou deixar de fazer pelo simples fato de estarem nesse momento da vida, sendo isto tão natural e que reflete inúmeros benefícios para quem pratica. Assim, este estudo tem por objetivo analisar a produção científica sobre a sexualidade na terceira idade e o papel do profissional enfermeiro enquanto educador em saúde.

\section{Desenvolvimento}

\subsection{Metodologia}

O presente artigo se trata de uma revisão narrativa realizada através da Biblioteca Virtual em Saúde e consultas aos bancos de dados ScientificElectronic Library Online (SciELO), Literatura Latino-Americana e do Caribe em Ciências da Saúde (Lilacs) e na Medical LiteratureAnalysisandRetrieval System Online (Medline).

Para composição desta revisão foram definidos alguns critérios de inclusão: artigos publicados recentemente e que estivessem disponíveis de forma completa, bem como a sua origem ser nacional, possuindo relação direta com o estudo. Logo, ao final dessa pesquisa foram escolhidos para desenvolver este trabalho apenas dez artigos.

Quadro 1 - Artigos capturados nas bases de dados descritas acima sobre Sexualidade na Terceira Idade no período de 2008-2015.

\begin{tabular}{|c|c|c|c|}
\hline Autores & Periódico & Ano & $\begin{array}{c}\text { Local de } \\
\text { Publicação }\end{array}$ \\
\hline $\begin{array}{c}\text { Almeida e } \\
\text { Lourenço }\end{array}$ & RBCEH & 2008 & $\begin{array}{c}\text { Rio Grande } \\
\text { do Sul }\end{array}$ \\
\hline $\begin{array}{c}\text { Almeida e } \\
\text { Lourenço }\end{array}$ & RBGG & 2010 & $\begin{array}{c}\text { Rio Grande } \\
\text { do Sul }\end{array}$ \\
\hline $\begin{array}{c}\text { Grandin, Sousa } \\
\text { e Lobo }\end{array}$ & CogitareEnferm & 2012 & Paraná \\
\hline Laurentino & $\begin{array}{c}\text { Rev. Bras. Ciên. } \\
\text { Envelh. Hum. }\end{array}$ & 2010 & $\begin{array}{c}\text { Rio Grande } \\
\text { do Sul }\end{array}$ \\
\hline
\end{tabular}




\begin{tabular}{|c|c|c|c|}
\hline Leite et al. & $\begin{array}{c}\text { Rev. Bras. Geriatr. } \\
\text { Gerontol. }\end{array}$ & 2010 & $\begin{array}{c}\text { Rio de } \\
\text { Janeiro }\end{array}$ \\
\hline Pottes et al. & Rev Bras Epidemiol & 2012 & São Paulo \\
\hline Ribeiro e Jesus & CogitareEnferm & 2012 & Paraná \\
\hline Risman & TextosEnvelhec. & 2015 & $\begin{array}{c}\text { Rio de } \\
\text { Janeiro }\end{array}$ \\
\hline Vaz e Nodin & AnálisePsicol. & 2015 & Portugal \\
\hline $\begin{array}{c}\text { Viana e } \\
\text { Madruga }\end{array}$ & Rev. Conexões. & 2013 & Paraná \\
\hline
\end{tabular}

Fonte: Dados da pesquisa.

\subsection{Dificuldades sexuais no envelhecimento}

Durante muito tempo a sexualidade foi vista pela sociedade como sinônimo de sexo estando ligada intrinsecamente à reprodução. Esse método tem origem pelas junções religiosas, políticas e sociais, tendo em vista o controle das atitudes ligadas ao prazer sexual inato que não fossem relacionados ao amor ou ao compromisso de uma relação, como o casamento. Dessa maneira, do que era natural se fez uma atitude automatizada entre os casais que ansiavam por constituir uma família (ALMEIDA; LOURENÇO, 2008).

A dinâmica do envelhecimento masculino é tendenciosa a desenvolver depressão, visto que eles têm maior dificuldade para compreender as próprias limitações da idade, entre essas as sexuais. Nesse aspecto, o uso de medicamentos abre uma nova expectativa em fazer o indivíduo resgatar a convicção que tinha na capacidade sexual em momentos passados da sua vida (RISMAN, 2015).

É bastante frequente que os homens idosos busquem por mulheres mais jovens como parceira sexual, para ele reflete o acréscimo da virilidade, todavia, repudia as mulheres idosas que se envolvem sexualmente com homens mais jovens. $\mathrm{Na}$ realidade, os idosos mantêm o impulso e a atividade sexual, mesmo havendo mudanças pelas alterações fisiológicas, expectativas socioculturais, problemas de saúde e medicações (LEITE et al., 2010).

Os problemas sexuais que acometem os idosos com maior frequência são: falta de conhecimento e/ou a timidez/ preconceito sobre o assunto, impotência nos homens e falta de lubrificação nas mulheres, falta de libido sexual por um dos parceiros, consequências secundárias oriundas do uso de medicamentos ou polimedicação, dores e comprometimentos físicos, demência por parte de um dos parceiros, óbito ou separação dos parceiros e o declínio da vida sexual, deixando a sexualidade disforme e a excitação insuficiente (MINAYO, 2010).

Com o aumento no número de pessoas idosas, cresceu da mesma forma os números de casos de Síndrome da Imunodeficiência Adquirida (AIDS) entre a população. As adversidades do envelhecimento e da AIDS no Brasil superam a questão da cultura e de restrição em centralizar e caracterizar o preconceito da sociedade pertinente ao sexo nessa idade (POTTES et al., 2012).

É importante que os idosos desfrutem da vida, entretanto, devem priorizar e cuidar da própria saúde. Realizar relações sexuais sem prevenção essencial remete aos mesmos riscos de contaminação de IST's como qualquer outra pessoa (BERNARDO; CORTINA, 2012). Sendo doenças adquiridas por vários tipos de agentes e transmitidas, sobretudo, por contato sexual sem o uso de preservativos (RIBEIRO; JESUS, 2012).

Sabendo-se que a população idosa está passando por crescente aumento, faz-se necessário educá-la, encorajando a prática sexual ativa de forma segura, pois os mesmos são tão susceptíveis em contrair infecções sexualmente transmissíveis quanto os mais jovens e, de certa forma, vulneráveis a maiores complicações. Dessa forma, cabe aos enfermeiros, enquanto educadores, visualizar os idosos de forma holística, afinal, são seres humanos e necessitam ser vistos como um todo para prevenir maiores agravos pela falta de informação.

\subsection{Qualidade da sexualidade no envelhecimento}

A qualidade de vida é o entendimento social formado com base nos padrões subjetivos de cada pessoa (bem-estar, prazer, realização pessoal), assim como objetivos, sendo a satisfação das necessidades vitais e necessidades criadas pelo nível de desenvolvimento econômico e social como principais referências (MINAYO, 2010).

Segundo a Organização Mundial de Saúde, o conceito de envelhecimento ativo é "o processo de aprimoramento de oportunidades para a saúde, participação e segurança, no sentido de aumentar a qualidade de vida durante o envelhecimento". A intenção é possibilitar ao idoso o envelhecimento com dignidade, favorecendo a sua independência e autonomia mediante presença ativa em nível social, cultural, espiritual e cívico, aproximando o conceito de saúde da OMS, que descreve o bem-estar completo em âmbito físico, psíquico e social.

Desse modo, as atividades físicas, alimentação correta, gerenciamento dos níveis de estresse, satisfação pessoal, prazer experimentado de diversas formas, contato social e profissional, o entretenimento, a vida amorosa e sexual, proporcionam condições para que o idoso viva de forma mais harmoniosa em termos individuais e sociais. Por esses motivos que se deve viver incansavelmente em prol de melhorias para a vida, pois não se tem como parar o tempo e controlar as coisas, já que as pessoas são impotentes para isso, resta viver com qualidade e isso vai além de um corpo funcional e saudável (VAZ; NODIN, 2015).

Vale salientar que a prática sexual é considerada um motivo que contribui para elevar a qualidade de vida. Dessa forma, o ato sexual é primordial, tanto quanto atividade física regular, que propicia aos idosos o desenvolvimento dessas atividades, diminuindo a possibilidade de se sentir inútil (GRANDIN; SOUSA; LOBO, 2012).

As atividades sexuais têm uma pluralidade de fins. Os ganhos que o indivíduo obtém dessa prática são diversos. Constantemente, o indivíduo idoso executa a atividade sexual com o propósito de remetê-lo de volta à juventude e aos 
instantes de extrema satisfação em seu passado. Pelo desejo de reacender as fantasias do seu passado viril. Todavia, esse desejo só é encontrado em idosos que atribuíram no decorrer da vida um valor positivo para atividade sexual, já os que repudiaram esse ato, utilizam a desculpa de que a velhice o fez abandonar a vida sexual ativa, por fim, segundo os autores, a vida sexual se estende em decorrência da felicidade obtida pelo exercício desta (VIANA; MADRUGA, 2013).

\section{$2.4 \mathrm{O}$ enfermeiro frente à sexualidade na terceira idade}

Frente à sexualidade na terceira idade, cabe ao enfermeiro o critério na percepção das alterações fisiológicas no idoso como método natural do envelhecimento, e como essas interferem na sexualidade e no vínculo terapêutico entre o profissional enfermeiro e o cliente (GRANDIN; SOUSA; LOBO, 2012).

Atualmente se fala sobre sexualidade, porém o diálogo sobre a prática sexual entre homens e mulheres durante o envelhecimento pouco é discutido e, em alguns momentos até menosprezado pelos profissionais de saúde e sociedade como um todo. Os profissionais de saúde não têm como prática, em seu atendimento, interrogar sobre os aspectos pertinentes à prática sexual, e menos ainda quando esses são idosos. Isso ocorre em razão de a atenção à saúde ser executada com o foco na queixa ou na doença (RIBEIRO; JESUS, 2012). Segundo os autores, não há identificação, na maioria das vezes, se as medicações utilizadas pelos clientes interferem na prática sexual. No senso comum, espera-se que tal prática desapareça com o decorrer da idade, por essa razão não há interrogação sobre a sexualidade na velhice. Desse modo, muitas vezes se deixa de abordar a temática com o cliente, deixando de prevenir doenças sexuais que acometem as pessoas nessa faixa etária, como a disfunção erétil, o vaginismo, dor durante a atividade sexual, uso inadequado de medicamentos e a prevenção da AIDS.

A sexualidade é temática delicada, de difícil conversação em um atendimento, porém é evidente que essa dificuldade está inserida na vida dos idosos. Sendo preocupantes os preconceitos e ideias a respeito da sexualidade na velhice, assim como o despreparo dos profissionais em lidar com esse assunto. O cuidado é o fundamento da ciência e da arte da enfermagem. Este cuidar está inserido na prática que vai além da assistência às necessidades inerentes ao ser humano no instante em que ele se encontra debilitado, é um compromisso com o cuidar que envolve o autocuidado, a autoestima, a cidadania do outro e da respectiva pessoa que cuida (GRANDIN; SOUSA; LOBO, 2012).

Em face ao contexto atual se nota a importância do enfermeiro na assistência ao idoso, expondo conhecimentos e explicando sobre a sexualidade, desse modo, estabelecendo vínculo com este. Tornando primordial o estabelecimento de uma relação de confiança para que o idoso exponha suas dúvidas e medos. Na atuação da enfermagem, as abordagens das diferenças do processo saúde-doença ocorrem quase como uma constante. Nessa concepção, a sexualidade deve ser considerada nas intervenções em conjunto com os idosos, na saúde e na doença como uma necessidade inerente ao ser humano. Ainda assim, muitos profissionais de saúde têm dificuldade em lidar com esse assunto, pois acreditam no mito de que os idosos não são mais ativos para a intimidade ou não possuem capacidade para relações íntimas (LAURENTINO, 2010). Em conformidade com o autor, o sexo é considerado fator que auxilia na qualidade de vida, pois sua prática pode ser comparada com o exercício físico.

\section{Conclusão}

Os idosos continuam tendo desejos, projeções, fantasias e afetos. O respeito e a conformação no intuito de se beneficiar ainda do ato desenvolvido sexualmente prevalecem na qualidade de vida, visto que oferece prazer e sensação de bem-estar. Ainda assim, a sociedade cria uma imagem dos indivíduos idosos, de que não necessitem de sexo, e com isso, muitos dispensam o prazer para não serem vistos aos olhos da sociedade como algo estranho.

Atualmente, a ciência proporciona aos idosos uma vida sexual ativa de forma mais agradável. Simultaneamente com o progresso da tecnologia se viabiliza que o ser humano evolua em seus pensamentos e aceite que a prática do sexo é algo natural e inerente também para quem não é jovem.

Espera-se que haja melhor aceitação da prática sexual durante a longevidade, visto que é um ato simples e que faz parte da saúde e bem-estar dos idosos. Contudo, é fundamental que a sociedade se interesse e se informe sobre esse assunto, mudando seu modo de pensar e tomando consciência enquanto familiares e profissionais de saúde que lidam continuamente com os idosos.

Visando o sexo ligado à saúde, e sendo o profissional enfermeiro tão importante e de referência no acompanhamento terapêutico e sexual dos idosos, como instrutor na educação em saúde sexual, juntamente com a equipe multiprofissional, minimizando as frustrações que se encaram hoje, para que as próximas gerações não sofram a mesma situação de preconceito atual. A enfermagem deve exercer e desempenhar conhecimentos acerca da sexualidade enquanto saúde e bemestar psicossocial, além de se fazer necessário compreender a sexualidade humana, em razão do cenário frequente que remete as transmissões de DST's e do vírus HIV por via sexual.

Durante a assistência, o profissional enfermeiro deve assegurar um ambiente que garanta a privacidade do cliente, respeitando a origem e a ideologia do idoso, deixando-o confortável para questionamentos e dúvidas.

Logo, o enfermeiro precisa entender as peculiaridades pertinentes aos idosos que fazem parte do cenário, no qual o profissional está inserido para poder contribuir, auxiliar e direcionar essa clientela a conviver com a sexualidade nessa etapa da vida, desenvolvendo ações de cuidados dirigidos à promoção da saúde e bem-estar, exemplificando suas 
limitações físicas, sociais e emocionais, adequando seus contentamentos às modificações fisiológicas que o corpo apresenta no transcorrer dos anos.

\section{Referências}

ALMEIDA, T.; LORENÇO, M. L. Envelhecimento, amor e sexualidade. $R B G G$, v.10, p.101-113, 2010.

ALMEIDA, T.; LORENÇO, M.L. Amor e sexualidade na velhice. $R B C E H$, v.5, p.130-140, 2008.

BERNARDO, R.; CORTINA, I. Sexualidade na terceira idade. Rev. Enferm. UNISA., v.13, n.1, p.74-78, 2012.

BRASIL. Organização Pan-Americana da Saúde. Envelhecimento ativo: uma política de saúde. Brasília: World Health Organization, 2005.

DUARTE Y. A.O.; DHIOGO, M.J.D. Atendimento domiciliar: o enfoque gerontológico. São Paulo: Atheneu, 2010.

GRANDIN, C.V.C.; SOUSA, A.M.M.; LOBO, J.M. A prática sexual e o envelhecimento. CogitareEnferm.,v.12, n.2, p.204$213,2012$.

LAURENTINO, N.R.S. Namoro na terceira idade e o processo de ser saudável na velhice. Rev. Bras. Ciênc. Envelh. Hum., p.5163, 2010.

LEITE, M.T. et al. Doenças sexualmente transmissíveis e HIV/ AIDS na opinião de idosos que participam de grupos de terceira idade. Rev. Bras. Geriatr. Gerontol., v.10, n.3, p.321-327, 2010.

MENDES, M.R.S.S.et al. A situação social do idoso no Brasil: uma breve consideração. Acta Paul Enferm., v.18, n. 4, 2005.

MINAYO, M.C.S. O desafio do conhecimento: pesquisa qualitativa em saúde. São Paulo: Hucitec, 2010.

PAPALÉO NETTO, M. Gerontologia. A velhice $e$ o envelhecimento em visão globalizada. São Paulo: Atheneu, 2010.

POTTES, F.A. et al. AIDS e envelhecimento: característica dos casos com idade igual ou maior que 50 anos em Pernambuco, de 1990 a 2000. Rev. Bras. Epidemiol., v. 10, n. 3, p. 338-351, 2012.

RIBEIRO, L.C.C.; JESUS, M.V.N. Avaliando a incidência dos casos notificados de AIDS em idosos no estado de Minas Gerais no período de 1999 a 2004. Cogitare Enferm., v.11, n.2, p.113116, 2012.

RISMAN, A. Sexualidade e terceira idade: uma visão históricocultural. Textos Envelhec., v.8, n.1, 2015.

RODRIGUES, R.A.P. et al. Política Nacional de Atenção ao Idoso e a contribuição da enfermagem. Texto Contexto Infere, v.16, n.3, p.536-534, 2012.

ROSENTHAL, S. H. Sexo depois dos 40. Rio de Janeiro: Ediouro, 2014.

SOUZA, M.S. Sexualidade na terceira idade. Rev. Educ. Meio Amb. Saúde, v.4, n.1, p.65-73, 2010.

VAZ, R.A.; NODIN, N.A importância do exercício físico nos anos maduros da sexualidade. Análise Psicol., v.3, p.329-339, 2015.

VIANA, H.B.; MADRUGA, V.A. Sexualidade, qualidade de vida e atividade física no envelhecimento. Rev. Conexões., v.6, p.222232, 2013. 